\title{
Statin Therapy in Metabolic Syndrome and Hypertension Post-JUPITER: What is the Value of CRP?
}

\author{
Sridevi Devaraj • David Siegel • Ishwarlal Jialal
}

Published online: 3 November 2010

(C) The Author(s) 2010. This article is published with open access at Springerlink.com

\begin{abstract}
Much evidence supports a pivotal role for inflammation in atherosclerosis. C-reactive protein (CRP), the prototypic marker of inflammation in humans, is a cardiovascular risk marker and may also promote atherogenesis. CRP levels are increased in metabolic syndrome and hypertension and confer increased risk of cardiovascular events in patients in these subgroups. Statins have been shown to lower low-density lipoproteins and CRP independently, and reduce cardiovascular events in subjects with and without metabolic syndrome and hypertension. In this review, we focus on the results from the primary prevention statin trial, Justification for the Use of statins in Primary prevention: an Intervention Trial Evaluating Rosuvastatin (JUPITER), which showed reductions in LDL, CRP, and cardiovascular events. Post-JUPITER, the new guidelines will now need to consider recommending high-sensitivity CRP testing to intermediate-risk metabolic syndrome patients and those with hypertension and intermediate risk so that we can better identify candidates at greater risk and reduce cardiovascular burden in these subjects with statin therapy.
\end{abstract}

Keywords CRP. Endothelial dysfunction .

Atherosclerosis · Inflammation · Monocytes · Statins ·

Metabolic syndrome $\cdot$ Hypertension

S. Devaraj $\cdot$ D. Siegel $\cdot$ I. Jialal $(\bowtie)$

Laboratory for Atherosclerosis and Metabolic Research,

UC Davis Medical Center,

4635 Second Avenue, Research 1 Building, Room 3000,

Sacramento, CA 95817, USA

e-mail: ishwarlal.jialal@ucdmc.ucdavis.edu

S. Devaraj · D. Siegel $\cdot$ I. Jialal

Department of Medicine, Veterans Affairs Medical Center,

Mather, CA, USA

\section{Introduction}

Much evidence supports a pivotal role for inflammation in all phases of atherosclerosis, from the initiation of the fatty streak to the culmination in acute coronary syndromes [1, 2]. C-reactive protein (CRP) is the prototypic marker of inflammation in humans.

High-Sensitivity CRP and Cardiovascular Risk

Numerous studies from various parts of the world have clearly established that CRP predicts future risk for cardiovascular disease (CVD) in apparently healthy persons, independent of established risk factors in the majority of studies. In the studies to date, CRP has been shown to predict myocardial infarction (MI), coronary artery disease (CAD) death, stroke, peripheral arterial disease, and sudden death [3-5]. In the Women's Health Study, Ridker et al. [6] have shown that CRP is additive to low-density lipoprotein (LDL) cholesterol and the Framingham 10-year risk score in predicting future CVD in healthy American women. Thus, based on these data, the American Heart Association and Centers for Disease Control and Prevention have issued a statement recommending that CRP be used as a risk marker for CVD in individuals with a Framingham risk score between $10 \%$ and $20 \%$ [3-5]. In their recommendations, CRP levels $<1 \mathrm{mg} / \mathrm{L}$ were considered low-risk, 1 to $3 \mathrm{mg} / \mathrm{L}$ as average risk, and $>3 \mathrm{mg} / \mathrm{L}$ as high risk for CVD. Conditions that have been associated with increased levels of CRP include adiposity, chronic inflammation, metabolic syndrome, type 2 diabetes, hypertension, chronic kidney disease, and sleep apnea [3-5].

CRP and Atherothrombosis

Although initial reports suggested a role of CRP as a surrogate of the underlying inflammatory process of 
atherothrombosis, accumulating evidence from in vitro and in vivo studies in clinical and experimental models strongly points towards a role of CRP as a proatherogenic factor (Table 1) $[3-6,7 \bullet \bullet]$.

\section{CRP and Endothelial Function}

An impressive amount of data now implicates CRP in inducing endothelial cell activation and dysfunction [3-6, $7 \bullet \bullet, 8]$. Earlier observations demonstrated that CRP levels correlated inversely with endothelial vasoreactivity. The most compelling data implicating CRP as a determinant of endothelial dysfunction were studies demonstrating that human CRP reduced basal and stimulated nitric oxide release from arterial and venous endothelial cells $[9,10]$. In human aortic endothelial cells (HAEC), CRP resulted in significant reduction in mRNA and protein for endothelial nitric oxide synthase (eNOS). Furthermore, CRP reduced eNOS activity (ie, conversion of L-arginine to L-citrulline) and bioactivity (secretion of cyclic guanosine 5'-monophosphate [cGMP]), in part through decreasing eNOS mRNA stability [9, 10]. CRP mediates uncoupling of eNOS via stimulation of NADPH oxidase and inhibition of GTP cyclohydrolase [11].

Another important product of endothelial cells is prostacyclin, a potent vasodilator, inhibitor of platelet aggregation, and inhibitor of smooth muscle cell proliferation. CRP in concentrations as low as $10 \mu \mathrm{g} / \mathrm{mL}$ resulted in a decrease in the release of the stable metabolite of prostacyclin, prostaglandin F-1 $\alpha$ (PGF-1 $\alpha$ ) in both human aortic and coronary artery endothelial cells, an effect that was mediated through increased nitration of prostacyclin synthase (PGIS) activity [12]. In addition, CRP has a direct effect on fibrinolysis via effects on increasing plasminogen activator inhibitor-1 (PAI-1) and decreasing tissue plasminogen activator tPA $[3-6,7 \bullet \bullet, 8]$. CRP activates Rho/Rho-kinase signaling, which in turn activates NF- $\mathrm{KB}$ activity, resulting in increased PAI-1 expression $[3-6,7 \bullet \bullet, 8]$.

\section{CRP and Monocytes}

Cermak et al. [13] were the first to demonstrate that CRP induced monocyte tissue factor (TF) secretion. Recently, we demonstrated that intraperitoneal administration of CRP (20 mg/kg) compared with human serum albumin (HuSA) increased superoxide anion release and TF activity from peritoneal macrophages in vivo $(P<0.01)$ [14]. This was confirmed using intrapouch administration of CRP $(25 \mu \mathrm{g} /$ $\mathrm{mL}$ ) compared with HuSA. In rat pouch macrophages in vivo, we showed that CRP induced TF antigen and activity was inhibited by inhibitors to NADPH oxidase. Furthermore, we demonstrated that inhibition of NF- $\mathrm{KB}$ activity resulted in significant inhibition of CRP-induced TF activity, and blockade of CD32 and CD64 reduced CRPinduced TF. Several investigators have demonstrated that CRP induces oxidative stress in vitro. We demonstrated that lower concentrations of CRP $(25 \mu \mathrm{g} / \mathrm{mL})$ in vivo induce superoxide anion release and that this is reversed by inhibition of protein kinase $\mathrm{C}$ (PKC) and NADPH oxidase. In rat pouch macrophages, we also demonstrated that the effects of CRP on superoxide anion release were inhibited via inhibition of ERK and c-Jun N-terminal kinase (JNK) but not p38 MAPK [14]. These effects are abrogated by blocking Fc receptors CD32 and CD64. Another important in vivo demonstration we made is the induction of myeloperoxidase activity in macrophages in vivo by human CRP administration [15]. CRP also induces proinflammatory cytokine release from human monocytes. A single report has shown increased $\mathrm{CD} 11 \mathrm{~b}$ expression on monocytes incubated with CRP, accompanied by increased adhesion of these monocytes to LPS-activated human umbilical vein endothelial cells $[3-6,7 \bullet \bullet, 8]$. We have shown that CRP increases monocyte-endothelial cell adhesion under shear stress and static conditions via increased NF-KB and increased intercellular adhesion molecule 1 (ICAM-1) and vascular cell adhesion molecule 1 (VCAM-1) expression $[3-6,7 \bullet \bullet, 8]$. We have also recently shown that CRP promotes macrophage colony-stimulating factor (M-

Table 1 Proatherogenic effects of C-reactive protein

\begin{tabular}{ll}
\hline Endothelium & Monocytes \\
\hline Increased VCAM, ICAM-1, E-selectin, MCP-1, monocyte adhesion & Increased tissue factor \\
Increased PAI-1, IL-8, CD40/CD40L, MMP-1, ET-1 & Increased M-CSF and proliferation \\
Decreased eNOS, prostacyclin, tPA, promotes endothelial dysfunction & Increased superoxide and myeloperoxidase \\
Increased proinflammatory cytokines & Decreased IL-10 \\
Impaired EPC number and function in vitro & Promotes oxLDL uptake and decreases cholesterol efflux \\
\hline
\end{tabular}

eNOS endothelial nitric oxide synthase, EPC endothelial progenitor cells, $E T$ endothelin, $I C A M$ intercellular adhesion molecule, $I L$ interleukin, $M C P$ monocyte chemotactic protein, $M$-CSF macrophage colony-stimulating factor, $M M P$ matrix metalloproteinase, ox $L D L$ oxidized low-density lipoprotein, $P A I$ plasminogen activator inhibitor, $t P A$ tissue plasminogen activator, VCAM vascular cell adhesion molecule 
CSF) release from human macrophages and induces macrophage proliferation; this effect appears to be mediated via NF$\mathrm{KB}$ activation and through the $\mathrm{Fc}$ receptors CD32 and CD64 [16]. In addition, we have demonstrated that in vivo administration of CRP promotes uptake of oxidized LDL (oxLDL) and intracellular cholesterol ester accumulation in rat macrophages in vivo [17]. The increased uptake of oxLDL by CRP was inhibited by pretreatment with antibodies to CD32, CD64, CD36, and fucoidin, suggesting uptake by both scavenger receptors and $\mathrm{Fc}$ receptor. Thus, it is clear that CRP is proatherogenic in monocyte-macrophages. Furthermore, CRP is present in foam cells in the atherosclerotic lesion and activates complement $[3-6,7 \bullet \bullet, 8]$.

CRP Promotes Atherothrombosis: Studies in Rats and Transgenic Mice

Additional evidence for an in vivo role of CRP in CVD stems from studies demonstrating the ability of CRP to induce MI in a rat coronary ligation model. These studies showed increase cerebral infarct size in rats following middle cerebral artery occlusion, and promotion of neointimal formation after balloon angioplasty $[3-6,7 \bullet \bullet, 8]$.

In the CRP transgenic mice, Danenberg et al. [18] showed that compared to wild-type mice in which CRP levels are undetectable, CRP levels increased to $18 \pm 6 \mathrm{mg} / \mathrm{L}$. Following injury to the femoral artery, there was complete thrombotic occlusion in the femoral artery in $75 \%$ of the human CRP transgenic mice when compared to $17 \%$ of the wild-type mice at 28 days $(P<0.05)$. Furthermore, arterial photochemical injury to the carotids shortened clot formation time in the human CRP transgenic mice compared to the wild-type mice. Although the human CRP transgene resulted in increased atherosclerotic lesion on an apolipoprotein $\mathrm{E}$ (apoE)-knockout background, it should be pointed out that the effect was only seen in male mice and was modest, questioning the validity of the mouse model. In two recent studies, transgenic expression of human CRP had no effect on development, progression, or severity of spontaneous atherosclerosis, or on morbidity or mortality, in male apoE-deficient C57BL/6 mice up to 56 weeks, despite deposition of human CRP and mouse complement component 3 in the plaques; again, there was no effect on atherosclerosis in the apoE*3-Leiden (E3L) transgenic mice carrying the human CRP gene [3-6, 7••, 8]. Also, Pepys et al. [19] have shown that a small molecule inhibitor (bis phosphatidylcholine) prevents the increase in infarct size following coronary ligation.

These studies indicate that CRP is not only a risk marker but may indeed promote atherogenesis. Two conditions where CRP levels are high are the metabolic syndrome (MetS) and hypertension, and high levels of CRP predict increased risk in these individuals [20].

\section{Metabolic Syndrome}

The metabolic syndrome (MetS) comprises a cluster of abnormalities, with insulin resistance (IR) and adiposity as central features [21]. Five diagnostic criteria have been identified by the National Cholesterol Education Program Adult Treatment Panel III (NCEP-ATP III), and the presence of any three features (central obesity, dyslipidemia [high triglycerides, low HDL], hypertension, and impaired fasting glucose) is considered sufficient to diagnose the syndrome. Approximately $35 \%$ of US adults have the MetS, and the prevalence increases with age [22]. MetS confers a two to fourfold increased risk for CVD [23] and fivefold increased risk of diabetes [23]. Low-grade chronic inflammation is a central feature of MetS and could contribute to increased risks of both CVD and diabetes in MetS. Circulating levels of several inflammatory molecules have been measured to assess their value in predicting CVD. The best characterized and well-standardized marker of these is CRP. Numerous studies have now confirmed that CRP levels are elevated in individuals with the MetS. Furthermore, it has been proposed that high-sensitivity CRP (hsCRP) be added as a clinical criterion for MetS and for creation of an hsCRP-modified CHD risk score [24].

Elevated hsCRP Levels and Increased Cardiovascular Risk in MetS

Evidence supporting the hypothesis that elevated CRP levels contribute to increased cardiovascular risk is now available from at least six major prospective studies. These studies include the Physicians Health Study (PHS), Women's Health Study (WHS), Atherosclerosis Risk in Communities (ARIC), Air Force/Texas Coronary Atherosclerosis Prevention Study (AFCAPS/TexCAPS) in the United States and the Monitoring Trends and Determinants in Cardiovascular Disease (MONICA) and Reykjavik studies from Europe [20, 23, 25]. Additionally, with regards to MetS, Yudkin et al. [26], in 107 non-diabetic subjects, conducted Z-score analyses and found a very significant correlation between inflammatory markers and several features of the MetS. CRP levels were shown to be strongly associated with insulin resistance calculated from the homeostatic model assessment (HOMA), blood pressure, low high-density lipoprotein (HDL), triglycerides, and to levels of the proinflammatory cytokines interleukin-6 (IL-6) and tumor necrosis factor (TNF). Body mass index (BMI) and insulin resistance were the strongest determinants of the inflammatory state. There is a linear relationship between the number of metabolic features and increasing levels of hsCRP. Furthermore, Festa et al. [27] from Insulin Resistance and Atherosclerosis Study (IRAS), showed that hsCRP was positively correlated with $\mathrm{BMI}$ ), waist circumference, blood pressure, triglycerides, 
cholesterol, LDL cholesterol, plasma glucose, and fasting insulin, and it was inversely correlated with HDL cholesterol and insulin sensitivity index. The strongest associations are observed between CRP levels, central adiposity, and insulin resistance. The largest study to date that examined the association between inflammation and the MetS was the Third National Health and Nutrition Examination Survey (NHANES) study [20, 23, 25]. In a representative sample of the US population (8570 participants $>20$ years of age), subjects with the MetS, defined using ATP III criteria, were more likely than those without the syndrome to have elevated levels of markers of inflammation such as CRP, fibrinogen, and leukocyte count. Thus, there appears to be a clear relationship between the numbers of metabolic features and increasing hsCRP levels. In addition, we have shown that CRP levels were equivalent to the ratio of highmolecular weight (HMW) adiponectin to CRP in predicting MetS using receiver-operating characteristic (ROC) analyses $[20,23,25]$. Thus, MetS is a proinflammatory state characterized by increased CRP levels.

Ridker et al. [6], in a large-scale population cohort of the Women's Health Study, evaluated the potential interrelationships between CRP, the MetS, and incident cardiovascular events. In the 8 -year prospective follow-up of 14,719 women in the WHS, an hsCRP of $>3 \mathrm{mg} / \mathrm{L}$ in patients with MetS predicted a greater age-adjusted relative risk for future cardiovascular events. Furthermore, they reported that at all levels of severity of the MetS, CRP added prognostic information with regard to subsequent risk of incident CVE and was additive to the Framingham Risk Score. Thus, it has been proposed that hsCRP be added as a clinical criterion for MetS and for creation of an hsCRP-modified coronary heart disease (CHD) risk score [24].

In the West of Scotland Coronary Prevention Study (WOSCOPS), in which 6447 men were followed for 4.9 years, an hsCRP level $>3 \mathrm{mg} / \mathrm{L}$ predicted greater cardiovascular risk in patients with the MetS in a multivariate model $[20,23]$. In the Framingham Offspring Study [23, 25], both CRP and MetS were independent predictors of new cardiovascular events but were not additive. In an Italian study, patients with MetS and CRP $>3 \mathrm{mg} / \mathrm{L}$ had higher incidence of both carotid and coronary artery disease $[20,23,25]$. It was demonstrated in the Nurses' Health Study and Health Professionals Follow-up Study (HPS) that although MetS was a strong predictor of CHD in both men and women, CRP was additive in men only $[23,25]$. In a smaller Japanese Study of 461 patients with acute MI, CRP levels were additive to MetS in predicting future major adverse cardiac events (MACE) [23, 25]. Collectively, all these studies support the hypothesis that increased CRP in the setting of MetS confers an increased risk of future cardiovascular events.
Additionally, a genome-wide association study has been performed recently among 6345 apparently healthy women in which 336,108 single nucleotide polymorphisms (SNPs) were evaluated as potential determinants of plasma CRP concentration $[23,25]$. Overall, seven loci that associate with plasma CRP at levels achieving genome-wide statistical significance were found. Two of these loci (GCKR and HNF1A) are suspected or known to be associated with maturity-onset diabetes of the young, one is a gene-desert region on $12 \mathrm{q} 23.2$, and the remaining four loci are in or near the leptin receptor protein gene, the apoE gene, the IL6 receptor protein gene, or the CRP gene itself. The protein products of six of these seven loci are directly involved in MetS, insulin resistance, beta-cell function, weight homeostasis, and/or premature atherothrombosis. Thus, it is concluded that common variation in several genes involved in metabolic and inflammatory regulation have significant effects on CRP levels, consistent with CRP's identification as a useful biomarker of risk for incident vascular disease and diabetes. Overall, it appears that in individuals with MetS, an elevated CRP confers a greater risk for cardiovascular events by its action on vascular cells, such as activation of monocytes and induction of endothelial cell dysfunction.

\section{Statins and CRP}

Several large clinical trials have demonstrated that statins (3-hydroxy-3-methylglutaryl coenzyme A [HMG-CoA] reductase inhibitors) decrease the incidence of cardiovascular events in both primary and secondary prevention [23, 25]. In these trials, statin therapy resulted in significant reduction in LDL cholesterol and it has been generally assumed that cholesterol reduction by statins is the predominant mechanism for the reduction in cardiovascular events. However, subgroup analyses of large clinical trials, such as WOSCOPS, Cholesterol and Recurrent Events (CARE), and HPS, have suggested that the beneficial effects of statins may extend to mechanisms beyond cholesterol reduction. Further evidence in support of the pleiotropic effects of statins is provided by angiographic trials that have demonstrated clinical improvements with statins far exceeding changes in the size of the atherosclerotic lesions. The Myocardial Ischemia Reduction with Acute Cholesterol Lowering (MIRACL) trial also demonstrated that statins were effective in reducing recurrent ischemic events in as soon as 16 weeks after an acute coronary event $[25,28]$. Although serum cholesterol was reduced $40 \%$, this time frame was probably too short for changes in vascular remodeling. In addition, in the Pravastatin or Atorvastatin Evaluation and Infection Therapy (PROVE-IT) study, individuals with hsCRP levels 
$<2 \mathrm{mg} / \mathrm{L}$ and $\mathrm{LDL}$ cholesterol $>1.8 \mathrm{mmol} / \mathrm{L}(70 \mathrm{mg} / \mathrm{dL})$ after statin therapy had cardiovascular event rates similar to those in individuals with LDL cholesterol $<1.8 \mathrm{mmol} / \mathrm{L}$ (70 $\mathrm{mg} / \mathrm{dL})$ but hsCRP $>2 \mathrm{mg} / \mathrm{L}$ after statin therapy $[25$, 28]. This suggests that lower inflammatory status confers as much protection as lower cholesterol levels in patients with acute coronary syndrome. Similarly, in the Reversal of Atherosclerosis with Aggressive Lipid Lowering (REVERSAL) trial, the benefits of atorvastatin in terms of reducing C-reactive protein levels and total atheroma volume in the coronary arteries exceeded those of pravastatin, even at similar cholesterol reductions, arguing for the pleiotropic effects of statins $[23,27]$. The clinical benefit of statin drugs used in these studies is manifest early in the course of lipid-lowering therapy well before plaque regression could occur, pointing to the pleiotropic effects of statins. Several prospective clinical trials have shown that statins lower hsCRP levels, and this effect appears to be independent of its effects on lipid lowering. Furthermore, we have shown that this is a class effect because simvastatin, pravastatin, and atorvastatin, at equivalent LDL cholesterol-lowering doses, significantly reduced hsCRP levels in patients with hyperlipidemia [29].

\section{Statins, CRP, and MetS}

Although several studies have shown beneficial pleiotropic effects of statins on lowering hsCRP, lowering other biomarkers of inflammation, and improving endothelial function [25, 28] (all of which may be important in reducing cardiovascular risk in MetS patients), very few clinical trials have examined the effects of the statins on cardiovascular events in patients with MetS (Table 2).

In the Scandinavian Simvastatin Survival Study (4S), subgroup analysis was performed on 3933 nondiabetic patients with clinically established CHD, serum total cholesterol level of 5.5 to $8.0 \mathrm{mmol} / \mathrm{L}$, and serum triglyceride level $\leq 2.5 \mathrm{mmol} / \mathrm{L}[23,24]$. Endpoints were total mortality, coronary mortality, major CHD event, myocardial revascularization, any CHD event, stroke, and any atherosclerotic event. Over the 5.4-year median followup period, simvastatin therapy produced similar significant reduction in serum lipid levels in 893 patients with MetS ( $23 \%$ of the nondiabetic subjects) and in 3040 patients without MetS. The relative risks (RR) of main endpoints in simvastatin-treated patients compared with placebo-treated patients with MetS were as follows: total mortality 0.54 (95\% CI, 0.36-0.82), coronary mortality $0.39(0.23-0.65)$, major CHD event $0.59(0.45-0.77)$, and any atherosclerotic event $0.69(0.56-0.84)$. The corresponding RRs in patients without MetS were $0.72(0.56-0.91), 0.62(0.45-0.84)$, $0.71(0.61-0.82)$, and $0.76(0.68-0.85)$. These studies showed that simvastatin resulted in substantial relative reduction in the risk of cardiovascular events in nondiabetic heart disease patients with or without MetS; however, the absolute benefit may be greater in patients with MetS because they are at a higher absolute risk.

In lower-risk primary prevention patients with and without MetS, lovastatin therapy in the AFCAPS/TexCAPS study also resulted in a similar significant reduction in major coronary events $[25,28]$. Indeed, in this study, it appeared that in those subjects that did not meet NCEPATP III criteria for treatment with a lipid-lowering agent, the benefit of statin therapy in reducing coronary events in MetS patients was only evident in those with CRP $>1 \mathrm{mg} / \mathrm{L}$. In subjects with CRP $<1 \mathrm{mg} / \mathrm{L}$, regardless of the presence of MetS, there was no benefit of statin therapy. Thus, in primary prevention in low-risk subjects, CRP may be an effective criterion to use to determine if statin therapy is warranted in subjects with MetS. The WOSCOPS was another primary prevention study which showed similar significant reduction in major coronary events in patients with and without MetS [23, 24].

A subanalysis of the Treating to New Targets (TNT) trial evaluated whether aggressive lowering of LDL cholesterol with high-dose atorvastatin provides superior cardiovascular benefit for patients with both heart disease and MetS compared to regular-dose atorvastatin $[25,28]$. In this subanalysis, there were 5584 MetS patients who were

Table 2 Statin trials and metabolic syndrome

\begin{tabular}{lll}
\hline Study & Statin & Endpoint \\
\hline 4S (secondary prevention & Simvastatin & Significant reduction in CVE in MetS \\
AFCAPS/TexCAPS (primary prevention) & Lovastatin & Significant benefit with hsCRP $>1 \mathrm{mg} / \mathrm{L}$ \\
WOSCOPS (primary prevention) & Simvastatin & Significant reduction in coronary events in MetS \\
TNT (secondary prevention) & Atorvastatin & Significant reduction in CVE in MetS with high-dose statin \\
JUPITER (primary prevention) & Rosuvastatin & Significant reduction in CVE, especially in those who achieved \\
& & hsCRP $<2$ mg/L with statin \\
\hline
\end{tabular}

CVE cardiovascular events, $h S C R P$ high-sensitivity C-reactive protein, MetS metabolic syndrome 
randomized to low-dose atorvastatin $(10 \mathrm{mg} / \mathrm{d} ; n=2820)$ or high-dose atorvastatin $(80 \mathrm{mg} / \mathrm{d} ; n=2764)$. The primary endpoint included the time to first major cardiovascular event (defined as death from CHD, nonfatal nonprocedurerelated MI, resuscitated cardiac arrest, or fatal or nonfatal stroke). In the patients with heart disease and MetS, mean on-treatment LDL cholesterol concentrations at 3 months were $99.3 \mathrm{mg} / \mathrm{dL}$ with low-dose atorvastatin and $72.6 \mathrm{mg} / \mathrm{dL}$ with high-dose atorvastatin. At a median follow-up of 4.9 years, major cardiovascular events occurred in $13 \%$ of patients receiving $10 \mathrm{mg} / \mathrm{d}$ atorvastatin compared with $9.5 \%$ of those receiving $80 \mathrm{mg} / \mathrm{d}$ of atorvastatin (hazard ratio [HR]= $0.71 ; 95 \% \mathrm{CI}, 0.61-0.84 ; P<0.0001)$. Irrespective of treatment assignment, significantly more patients with MetS $(11.3 \%)$ had a major cardiovascular event than those without MetS (8.0\%; HR=1.44; 95\% CI, 1.26-1.64; $P<0.0001)$. This increased risk was significantly reduced by $29 \%$ in those on intensive therapy with high-dose atorvastatin beyond that achieved with regular-dose atorvastatin in the subgroup of MetS patients without diabetes, whereas only $8.2 \%$ persons receiving $80 \mathrm{mg} / \mathrm{d}$ of atorvastatin had a primary event, a much larger number (ie, $11.6 \%$ receiving $10 \mathrm{mg} / \mathrm{d}$ of atorvastatin) experienced the primary event. These findings represent a $30 \%$ relative reduction in the risk of a major cardiovascular event in favor of the highdose atorvastatin subset (relative risk of 0.70 ; $95 \%$ CI, $0.57-$ $0.84 ; P=0.0002)$. These results argue for aggressive treatment of heart disease patients with MetS with high-dose statin therapy, which was also safe. However, this study failed to study subjects with MetS alone without heart disease, and thus it is unclear if these results can be extrapolated to MetS subjects without heart disease.

Furthermore, because all of these trials were post hoc analyses, abdominal obesity was not examined with the accepted measure of the waist circumferences, instead a BMI $>28$ to $30 \mathrm{~kg} / \mathrm{m}^{2}$ was used. Also, the different trials used different definitions of the MetS, especially with regards to the cut-offs of fasting glucose. Thus, future statin trials in MetS subjects should assess the effects of statin therapy on all features of the MetS as identified by the modified NCEP-ATP III criteria.

\section{The JUPITER Trial, CRP, and MetS}

The Justification for the Use of statins in Primary prevention: an Intervention Trial Evaluating Rosuvastatin (JUPITER) trial addressed the hypothesis that men and women without overt CVD, with an LDL cholesterol below the current threshold for treatment, an elevated CRP would benefit from statin therapy. JUPITER enrolled 17,802 apparently healthy middle-aged men $(\geq 50$ years $)$ and women ( $\geq 60$ years) from 1315 sites in 26 countries.
Patients were free of diabetes and heart disease, with normal LDL levels $(<130 \mathrm{mg} / \mathrm{dL})$ but high hsCRP levels $(\geq 2.0 \mathrm{mg} / \mathrm{L}$ [median of $4.2 \mathrm{mg} / \mathrm{L}$ ]). Participants were randomly assigned to either $20 \mathrm{mg} / \mathrm{d}$ of rosuvastatin or placebo. Enrollees were followed for a primary composite endpoint of MI, hospitalization for unstable angina, heartrelated death, arterial revascularization, or stroke. Although planned as a 4-year study, the trial was stopped after a median follow-up time of 1.9 years based upon advice from an independent monitoring board and study steering committee $[30,31 \bullet \bullet]$.

Rosuvastatin $(20 \mathrm{mg} / \mathrm{d})$ lowered CRP levels by $37 \%$ (down to a 12-month mean of $2.2 \mathrm{mg} / \mathrm{L}$ ), lowered LDL by $50 \%$ (down to a 12 -month mean of $55 \mathrm{mg} / \mathrm{dL}$ ), and sharply decreased the number of cardiovascular events and allcause mortality. At the end of 1.9 years, rosuvastatin therapy significantly lowered the primary composite endpoint by $44 \%$ compared with placebo. In particular, there was a $55 \%$ lowering of nonfatal MI, $48 \%$ fall in the risk of nonfatal stroke, and a $47 \%$ fall in the risk of serious cardiac events $[30,31 \bullet \cdot]$. The reduction in vascular risk was greater in JUPITER than in previous statin trials. Furthermore, it was later shown that absolute risk reductions associated with rosuvastatin treatment were greater among those $\geq 70$ years of age than among the younger group. For the primary endpoint, there was an event rate reduction of 0.77 versus 0.52 per 100 person-years in the older versus younger group. This would mean one-third fewer people needing treatment to prevent an event over 5 years, at a number-needed-to-treat (NNT) of 19 in the elderly group versus 29 in the younger group.

In the subgroup of MetS subjects (41\% of subjects), there was a similar significant benefit as in those without MetS. Furthermore, in subjects who achieved on treatment $\mathrm{LDL}<1.8 \mathrm{mmol} / \mathrm{L}$ and $\mathrm{hsCRP}<2 \mathrm{mg} / \mathrm{L}$, the reduction in events was significantly greater (65\% reduction). In this trial, it is important to note that in the rosuvastatin group, achieved hsCRP levels were predictive of event rates, irrespective of the target LDL or apoB to apoA-1 ratio [30, $31 \bullet \cdot$. These data indicate that in subjects with and without MetS, reduction in events with statin therapy can be monitored by reductions in both LDL cholesterol and hsCRP.

The recent Canadian primary prevention guidelines endorse the use of statin therapy among individuals at intermediate risk who have elevated levels of hsCRP [32 • ]. In JUPITER, 6091 participants (2525 women and 3566 men) had baseline estimated 10-year Framingham risks of 5\% to $10 \%$, and 7340 participants (1404 women and $5936 \mathrm{men}$ ) had baseline estimated Framingham risk of $11 \%$ to $20 \%$. In these two subgroups, relative risk reductions consistent with the overall trial treatment effect were observed $(\mathrm{HR}=0.55 ; 95 \%$ CI, $0.36-0.84 ; 5$-year NNT $=40 ; P=0.005$ for those with $5 \%$ 
to $10 \%$ risk; hazard ratio $=0.51 ; 95 \%$ CI, $0.39-0.68 ; 5$-year NNT $=18 ; P<0.0001$ for those with $11 \%$ to $20 \%$ risk). Consistent with recent evidence-based Canadian Cardiovascular Society guidelines for primary prevention, the JUPITER trial demonstrated that $20 \mathrm{mg} / \mathrm{d}$ of rosuvastatin significantly reduces major cardiovascular events among men and women with elevated hsCRP and intermediate $(10 \%$ to $19 \%) 10$-year risk.

\section{Summary of Statin Therapy in MetS}

JUPITER will undoubtedly change the way primary prevention patients with low cholesterol levels are treated. If data from the placebo group in the JUPITER trial are extrapolated over 10 years, the 10-year risk of CHD events (MI and cardiovascular death) in the placebo group is about $6 \%$. For patients with a Framingham risk score of $10 \%$ to $20 \%$, the updated ATP III guidelines from 2004 recommend an LDL goal of $<130 \mathrm{mg} / \mathrm{dL}$ with an optional goal of $<100 \mathrm{mg} / \mathrm{dL}$. However, for patients with cardiovascular risk factors and Framingham risk score $<10 \%$, ATP III recommends considering pharmacologic therapy only if the LDL is $>160 \mathrm{mg} / \mathrm{dL}$. Although rosuvastatin was clearly beneficial in the JUPITER population, most of the patients in the JUPITER study would not have received statin therapy according to the current ATP III guidelines. Given the benefits of statin therapy seen in JUPITER, it would be reasonable to measure hsCRP levels in asymptomatic women 50 years and older and asymptomatic men 60 years and older with MetS in whom the decision to treat with statin therapy is unclear and to consider statin therapy for those with elevated hsCRP levels ( $>2 \mathrm{mg} / \mathrm{L}$ ). Also, it would be prudent to initiate statin therapy in individuals at intermediate risk (10\%-19\% Framingham risk score) with elevated levels of hsCRP.

\section{Statin Therapy in Hypertension}

Cardiovascular risk factors frequently occur together, and their combined unfavorable effects are generally greater than the sum of their individual components. In the MetS, hypertension frequently co-exists with dyslipidemia, obesity, and abnormalities of glucose metabolism (particularly insulin resistance). Indeed, hypertension and dyslipidemia are the most important cardiovascular risk factors in patients for both the primary and secondary prevention of CVD, and these risk factors occur together more than $50 \%$ of the time [33]. A recent analysis of NHANES 2003-2004 showed that the prevalence of hypertension ranged from $23.1 \%$ in those without CVD to $51.8 \%$ to $81.1 \%$ in those with cardiovascular co-morbidities (chronic kidney disease
$81.1 \%$; diabetes $76.8 \%$; peripheral artery disease $73.7 \%$; coronary artery disease $73.0 \%$; congestive heart failure $71.4 \%$; stroke $69 \%$; metabolic syndrome $61.5 \%$; dyslipidemia 51.8\%) [34].

The large benefit achieved in decreasing cardiovascular events with statin treatment in patients with a wide range of cholesterol levels cannot be attributed to only their cholesterol-lowering effects. This conclusion is based partly on the rapidity of the decrease in cardiovascular events after statin initiation. The benefits of these effects, independent of their lipid-lowering actions, have been referred to as the pleiotropic effects of statins. Some of these effects include modification of the toxic effects of circulating LDL cholesterol on vascular endothelium, vascular smooth muscle cell hypertrophy and proliferation, and fibrin deposition [35].

Statins, Endothelium and Hypertension

The vascular endothelium is a dynamic endocrine structure that regulates contractile, secretory, and mitogenic activities in the vessel wall [36]. It also affects hemostatic processes within the vascular lumen. Endothelial dysfunction is present in patients with a variety of cardiovascular risk factors, including hypertension, diabetes mellitus, dyslipidemia, and abnormalities of glucose metabolism. Statin therapy may have a beneficial effect on endothelial dysfunction. Statins may restore endothelial function by increasing the bioavailability of nitric oxide (NO), reducing oxidative stress, and inhibiting inflammatory responses [36]. Of interest, statins exhibit their effects on vascular endothelium quite early, generally within $24 \mathrm{~h}$ in the course of treatment. This may be mediated by the effect of statins on $\mathrm{NO}$ and the reduction of oxidative stress [37].

Epidemiology and Pathophysiology

Hypertension and dyslipidemia are frequently thought of as different risk factors, but they are both associated with markers of inflammation and oxidative stress that point to a common metabolic pathway resulting in the problems associated with these disorders. Epidemiologic studies have demonstrated that the presence of familial combined hyperlipidemia was increased 10 -fold in individuals with essential hypertension [38]. Metabolic abnormalities (eg, dyslipidemia and insulin resistance) are associated with increased risk for the subsequent development of hypertension. These frequently co-exist in individuals to a higher degree than expected based upon their individual prevalence. Additionally, multiple abnormalities that predispose to the development of hypertension are demonstrated in the normotensive children of hypertensive adults. In the Genetic Epidemiology Network of Arteriopathy (GENOA) 
study, a higher than expected prevalence of dyslipidemia was found in hypertensive patients [39]. In addition to NO, endothelial vasoreactivity is dependent on a number of other molecules. Inhibition of the production of potent endothelial-derived vasoconstrictors such as endothelin (ET-1) results in improvement of blood flow. Statins have been shown to decrease the expression of ET-1, mRNA, and ET-1 synthesis in bovine aortic endothelial cells in vitro [40].

The vascular renin-angiotensin-aldosterone system (RAAS) is involved in the early stages of endothelial dysfunction and inflammation, and is important in the development of hypertension. It may also promote a cascade of cellular and molecular events leading to the formation of atherosclerosis and plaque instability. Increased cholesterol is also associated with an overexpression of angiotensin II type one receptors in experimental models as well as in humans [41]. This may lead to an increase of angiotensin II-induced blood pressure elevation [36, 41]. There is some evidence that statins may partly restore the increased sensitivity to angiotensin II in healthy young adults with isolated elevated cholesterol and down-regulate AT-1 receptor expression. In a rat model, it was found that dietary lipids may affect sodium handling and the development of hypertension. Cholesterol may decrease sodium efflux along the nephron, resulting in a reduction in the fractional clearance of sodium and favoring in its retention. Interestingly, atorvastatin has been reported to increase the urinary secretion of sodium in conjunction with an increase in renal secretion of uric acid.

As noted above, hypertension and dyslipidemia are both characterized by increased levels of circulating markers of inflammation and oxidative stress. Hypertension induces endothelial dysfunction by a variety of mechanisms in addition to inflammation and oxidative stress. Structural alterations of the vasculature may occur following exposure to sheering forces with elevated blood pressure, with resulting progressive deposition of collagen within the vascular wall. This deposition of collagen may result in physiologic abnormalities of the wall, with increased vascular stiffness and reduction in compliance. In addition, the induced structural alterations in the vessels may result in pathologic alterations in vascular physiology. Hypertensive endothelial dysfunction may result in the release of $\mathrm{NO}$ coupled with the resulting decrease in the capacity for vasodilation.

\section{CRP and Hypertension}

As noted above, inflammation is felt to have a major role in the development of hypertension. In this regard, CRP levels have been shown to be associated with hypertension [42].
Elevated CRP may contribute to endothelial dysfunction by reducing the production of $\mathrm{NO}$, resulting in vasoconstriction. Additionally or alternatively, CRP may be a proatherosclerotic factor. In one study, it was found that an elevated CRP value preceded new-onset hypertension at an early stage among an elderly healthy population [43]. Alternatively, elevated blood pressure could induce a vascular inflammatory state, resulting in an elevated CRP [44].

Regardless of whether an elevated CRP is a cause or an effect of hypertension, the association between an elevated CRP and cardiovascular disease is clear. In the WHS, CRP was found to be a strong independent predictor of the development of hypertension [6, 24]. Analysis of 15,215 healthy women over the age of 45 years found a step-wise association between blood pressure levels and median levels of CRP; this step-wise association was noted for both systolic and diastolic blood pressure. In risk factoradjusted models, controlling for other cardiovascular risk factors, women with blood pressure $<120 /<75 \mathrm{~mm} \mathrm{Hg} \mathrm{had}$ a mean level CRP of $1.33 \mathrm{mg} / \mathrm{L}$ in comparison to women with blood pressure $\geq 160 / \geq 95 \mathrm{~mm} \mathrm{Hg}$, who had a mean CRP level of $1.84 \mathrm{mg} / \mathrm{L}$. Both CRP and blood pressure were strong independent predictors of future cardiovascular events. Those individuals with CRP levels in the top quartile had a twofold increased risk of developing elevated blood pressure compared to those with the CRP levels in the lowest quartile. Additional analysis showed that among women with either high or low blood pressure, survival was worse if CRP level were elevated $\geq 3 \mathrm{mg} / \mathrm{L}$.

There are several possible mechanisms by which CRP levels may cause increasing blood pressure. Hypertension may directly promote inflammation of blood vessels. Subjects with elevated blood pressure demonstrate increased production of reactive oxygen species as well as increased oxidative stress. CRP may also decrease NO production by endothelial cells [8-11]. In hypertensive patients, elevated CRP levels are associated with arterial stiffness. Increased vessel stiffness has been found to be a predictor of elevated blood pressure in normotensive offspring of hypertensive individuals. Aortic stiffness has also been shown to be a strong independent predictor of cardiovascular illness in hypertensive patients. In summary, by increasing ET-1 and IL-6 and reducing NO production, CRP may change the vascular system toward a proinflammatory and vasoconstrictive state with increased arterial stiffness, resulting in hypertension [8-11].

\section{Effect of Statins on Blood Pressure Levels}

The evidence for the blood pressure-lowering effects of statins in humans has been mixed, but the preponderance of data indicates that there is a reduction of blood pressure 
after statin use in the majority of individuals. The effect of statin therapy on blood pressure is generally modest, with reduction in systolic blood pressure of 3 to $5 \mathrm{~mm} \mathrm{Hg}$ and diastolic blood pressure of 1 to $3 \mathrm{~mm} \mathrm{Hg}$. The effect of statins on blood pressure appears to be most pronounced in individuals with higher and more difficult to control hypertension.

In the Brisighella Heart Study, 1356 hypercholesterolemic individuals were randomly assigned to a low-fat diet, cholestyramine, gemfibrozil, or simvastatin for five years. Participants were divided into four quartiles according to systolic blood pressure. A significant decrease in blood pressure was noted in the two upper quartiles of systolic blood pressure and was greater in those treated with lipidlowering drugs. Notably, the blood pressure reduction was greatest in patients treated with simvastatin despite a comparative reduction in LDL cholesterol [45].

A meta-analysis of all studies that reported blood pressure data during statin treatment included 20 randomized controlled trials and 828 patients [46]. Treatment with statins during the studies ranged from 1 to 12 months. Systolic blood pressure was significantly lower in patients treated with statins than in patients on placebo or on another lipid-lowering medication. The average decrease of $1.9 \mathrm{~mm} \mathrm{Hg}$ was modest. However, the effect on blood pressure was more pronounced when the analysis was restricted to studies with a baseline blood pressure of $>130 \mathrm{~mm} \mathrm{Hg}$. Here, the average change is systolic blood pressure was $4.0 \mathrm{~mm} \mathrm{Hg}$. Diastolic blood pressure was also lowered on average by $0.9 \mathrm{~mm} \mathrm{Hg}$ in all studies but by $1.2 \mathrm{~mm} \mathrm{Hg}$ in studies with patients with a diastolic blood pressure of $>80 \mathrm{~mm} \mathrm{Hg}$.

The benefit of lowering blood pressure and cholesterol was evaluated in the Antihypertensive and Lipid-Lowering Treatment to Prevent Heart Attack Trial (ALLHAT) [47]. One arm of ALLHAT was designed to determine whether pravastatin, compared with usually care, would reduce allcause mortality in 10,355 patients with hypertension and moderate hypercholesterolemia along with at least one other CHD risk factor. All-cause mortality was similar between the $40-\mathrm{mg} / \mathrm{d}$ pravastatin group and the usual-care group. These results could be attributed to the small differences in total cholesterol $(9.6 \%)$ and LDL cholesterol $(16.7 \%)$ between the two groups.

In the Anglo-Scandinavian Cardiac Outcomes TrialLipid Lowering Arm (ASCOT-LLA) trial, extra antihypertensive drug use in the placebo group may have obscured the atorvastatin blood pressure-lowering effect [48]. In this study, 19,342 men and women with hypertension and at least three other cardiac risk factors were randomized to different antihypertensive drug groups. A total of 10,305 of these patients with normal or slightly elevated total cholesterol were randomized to atorvastatin $(10 \mathrm{mg} /$ day $)$ or placebo. The atorvastatin arm was stopped prematurely at 3.3 years because of a significant reduction in the primary endpoint $(-36 \% ; P=0.005)$. In addition to the benefit on total cholesterol and LDL-C, the atorvastatin group had a decrease in blood pressure at 18 months of $0.7 \mathrm{~mm} \mathrm{Hg}$.

A cross-sectional study of 2584 hypertensive patients with no known CVD from the NHANES 1999 to 2002 compared people not receiving a statin medication with those who were [49]. Significantly more statin users had blood pressure $<140 / 90 \mathrm{~mm} \mathrm{Hg}(52.2 \%$ vs $38.0 \%)$, but these individuals were also more likely to comply with a low-salt diet use, use antihypertensive medication, and be nonsmokers. The Plaque Hypertension Lipid Lowering Italian study (PHYLLIS) was the largest study using office determination of blood pressure and included 508 hypertensive hypercholesterolemic patients treated with hydrochlorothiazide or fosinopril for 2.6 years. There was no additional effect on blood pressure when pravastatin $(20 \mathrm{mg} / \mathrm{d})$ was added.

Results of studies of statins on blood pressure during ambulatory blood pressure monitoring (ABPM) have generally found that there is a modest reduction of blood pressure with the use of statins. In one study in hypertensive patients with elevated cholesterol, statin therapy resulted in a non-significant tendency to reduce casual systolic and diastolic blood pressures, but average daytime systolic and diastolic blood pressures dropped significantly [50]. There was not a significant change in night time blood pressure or in circadian blood pressure profiles. In another study that compared the effects of adding atorvastatin or diet to antihypertensive agents in hypertensive hypercholesterolemic patients, there was a reduction in systolic and diastolic blood pressures with the statin. Adding a moderate dose of atorvastatin to antihypertensive agents in hypercholesterolemic patients with resistant hypertension resulted in a significant reduction in systolic and diastolic blood pressures than that found with a change in diet alone. The results of statins on ABPM have not always yielded blood pressuring-lowering effects. In a study comparing the effects of valsartan alone with valsartan plus simvastatin on 24-hour APBM, there was no difference between the two experimental groups.

There are a number of difficulties in the clinical trial assessment of the antihypertensive effects of statins. In many of the clinical trials described above, the effect of statins on blood pressure was not the primary endpoint of the trial. Patient populations have been heterogeneous, including dyslipidemic patients in studies of both normotensive and hypertensive subjects. Thus, the effect of statins on blood pressure could have been masked by other antihypertensive medications given to some of the subjects. In many of the studies, different hypertensive drugs were 
used, as well as different statins at different doses. Antihypertensive medications have different pharmacologic effects, including differential effects on endothelial function. This could lead to different interactions with statin therapy in terms of blood pressure lowering effects.

In JUPITER, 10,208 of the total 17,802 patients had hypertension. Rosuvastatin therapy significantly decreased the primary endpoint of nonfatal MI, stroke, revascularization, angina, and CVD death similarly in those with and without hypertension, with a concomitant reduction in LDL cholesterol and hsCRP levels [30, 31・•].

In addition, hypertension results in chronic kidney disease (CKD). In the post hoc analyses of JUPITER in those with moderate $\mathrm{CKD}$, rosuvastatin was associated with a significant $45 \%$ reduction in MI, angina, stroke, CVD death, and a $44 \%$ reduction in all-cause mortality [51 • $]$. LDL and hsCRP reductions were similar in those with and without CKD. Furthermore, median estimated glomerular filtration rate (eGFR) improved in those that received rosuvastatin compared to placebo.

\section{Summary of Statin Therapy in Hypertension}

Statins appear to exert a modest, but clinically relevant, antihypertensive effect. However, their benefit in hypertensive patients is considerably beyond that which would be predicted based on their effects on lipids as well as on blood pressure. This appears to be secondary to statins' effects on proatherosclerotic processes that are common to many cardiovascular risk factors including hypertension as well as dyslipidemia. These include the beneficial effects of statins on inflammation, endothelial function, oxidative stress, fibrinolytic parameters, and platelet function as well as on a variety of other effects involved in the atherosclerotic process. CRP, a marker of systemic inflammation, has received major attention for its role in the development and maintenance of hypertension as well as of CVD. In addition to other actions, by increasing ET-1 and IL-6 and reducing NO production, CRP may change the vascular system towards a proinflammatory and vasoconstrictive state with increased arterial stiffness, resulting in hypertension. Statins have been shown to lower CRP levels in a dosedependent fashion and thus may exert part of their benefits by several parameters involved in the atherosclerotic process.

\section{Conclusions}

Prospective randomized trials of MetS patients identified using NCEP-ATP III criteria that examined benefits on LDL cholesterol, measures of endothelial function and inflammation, especially hsCRP, and using such criteria to determine the contribution to reduction in cardiovascular events in patients with MetS, would prove very instructive. Until then, all MetS patients who have a Framingham risk score of $10 \%$ to $20 \%$ and elevated hsCRP should be treated with statins to reduce the burden of subsequent CVD. Increased CRP levels integrate a myriad of metabolic abnormalities such as increased adiposity, diabetes, smoking, end-stage renal disease, hypertension, and MetS. The new NCEP-ATP III guidelines will now need to consider recommending hsCRP testing to intermediate-risk MetS patients and those with hypertension and intermediate risk so that we can better identify candidates at greater risk and reduce cardiovascular burden in these patients with statin therapy.

Acknowledgment Grant Support: NIH K24AT00596, NIH RO1HL74360

Disclosure Sridevi Devaraj reports no potential conflict of interest relevant to this article. David Siegel reports no potential conflict of interest relevant to this article. Ishwarlal Jialal reports no potential conflict of interest relevant to this article.

Open Access This article is distributed under the terms of the Creative Commons Attribution Noncommercial License which permits any noncommercial use, distribution, and reproduction in any medium, provided the original author(s) and source are credited.

\section{References}

Papers of particular interest, published recently, have been highlighted as:

•- Of major importance

1. Ross R Atherosclerosis: an inflammatory disease's Engl J Med 1999; 340:115-26.

2. Hansson GK, Libby P The immune response in atherosclerosis: a double-edged sword. Nat Rev Immunol 2006; 6:508-19.

3. Verma S, Szmitko PE, Ridker PM C-reactive protein comes of age. Nat Clin Pract Cardiovasc Med 2005; 2:29-36.

4. Verma S, Devaraj S, Jialal I Is C-reactive protein an innocent bystander or proatherogenic culprit? C-reactive protein promotes atherothrombosis. Circulation 2006; 113:2135-50.

5. Ridker PM High-sensitivity C-reactive protein and cardiovascular risk: rationale for screening and primary prevention. Am J Cardiol. 2003;92:17K-22K.

6. Ridker PM, Buring JE, Shih J, Matias M, Hennekens CH. Prospective study of C-reactive protein and the risk of future cardiovascular events among apparently healthy women. Circulation. 1998;98:731-3.

7. •- Devaraj S, Singh U, Jialal I. The evolving role of C-reactive protein in atherothrombosis. Clin Chem. 2009;55:229-38. This review article details the proatherogenic effects of CRP on different cells in atherosclerosis.

8. Devaraj S, Yun JM, Adamson G, et al. C-reactive protein impairs the endothelial glycocalyx resulting in endothelial dysfunction. Cardiovasc Res. 2009; 84(3):479-84 
9. Hein TW, Singh U, Vasquez-Vivar J, et al. Human C-reactive protein induces endothelial dysfunction and uncoupling of eNOS in vivo. Atherosclerosis. 2009;206(1):61-8.

10. Valleggi S, Devaraj S, Dasu MR, Jialal I. C-reactive protein adversely alters the protein-protein interaction of the endothelial isoform of nitric oxide synthase. Clin Chem. 2010;56 (8): $1345-8$.

11. Singh U, Devaraj S, Vasquez-Vivar J, Jialal I. C-reactive protein decreases endothelial nitric oxide synthase activity via uncoupling. J Mol Cell Cardiol. 2007 ;43(6):780-91.

12. Venugopal SK, Devaraj S, Jialal I. C-reactive protein decreases prostacyclin release from human aortic endothelial cells. Circulation 2003;108: 1676-8.

13. Cermak J, Key NS, Bach RR, et al. C-reactive protein induces human peripheral blood monocytes to synthesize tissue factor. Blood 1993;82:513-20.

14. Devaraj S, Dasu MR, Singh U, et al. C-reactive protein stimulates superoxide anion release and tissue factor activity in vivo. Atherosclerosis. 2009;203(1):67-74.

15. Singh U, Devaraj S, Jialal I. C-reactive protein stimulates myeloperoxidase release from polymorphonuclear cells and monocytes: implications for acute coronary syndromes. Clin Chem 2009; 55:361-4.

16. Devaraj S, Yun JM, Duncan-Staley C, Jialal I. C-reactive protein induces M-CSF release and macrophage proliferation. J Leukoc Biol. 2009;85(2):262-7.

17. Singh U, Dasu MR, Yancey PG, Afify A, Devaraj S, Jialal I. Human C-reactive protein promotes oxidized low density lipoprotein uptake and matrix metalloproteinase-9 release in Wistar rats. J Lipid Res 2008; 49:1015-23.

18. Danenberg HD, Szalai AJ, Swaminathan RV, et al. Increased thrombosis after arterial injury in human C-reactive proteintransgenic mice. Circulation 2003; 108:512-5.

19. Pepys MB, Hirschfield GM, Tennent GA, et al. Targeting Creactive protein for the treatment of cardiovascular disease. Nature (Lond) 2006; 440: 1217-21.

20. Devaraj S, Singh U, Jialal I. Human C-reactive protein and the metabolic syndrome. Curr Opin Lipidol. 2009;20(3):182-9.

21. Eckel RH, Grundy SM, Zimmet PZ.The metabolic syndrome. Lancet. 2005; 365,1415-1428.

22. Alberti KG, Eckel RH, Grundy SM, et al. International Diabetes Federation Task Force on Epidemiology and Prevention; Hational Heart, Lung, and Blood Institute; American Heart Association; World Heart Federation; International Atherosclerosis Society; International Association for the Study of Obesity. Harmonizing the metabolic syndrome: a joint interim statement of the International Diabetes Federation Task Force on Epidemiology and Prevention; National Heart, Lung, and Blood Institute; American Heart Association; World Heart Federation; International Atherosclerosis Society; and International Association for the Study of Obesity. Circulation. 2009;120(16):1640-5.

23. Eckel RH, Alberti KG, Grundy SM, Zimmet PZ. The metabolic syndrome. Lancet. 2010;375(9710):181-3.

24. Ridker PM, Wilson PW, Grundy SM. Should C-reactive protein be added to metabolic syndrome and to assessment of global cardiovascular risk? Circulation. 2004;109:2818-2825.

25. Jialal I, Devaraj S. Statin therapy for the metabolic syndrome: the evidence base. Metab Syndr Relat Disord. 2009;7(5):393-6.

26. Yudkin JS, Kumari M, Humphries SE, et al. Inflammation, obesity, stress and coronary heart disease: is interleukin-6 the link? Atherosclerosis. 2000; 148:209-214.

27. Festa A, D'Agostino R Jr, Howard G, et al. Chronic subclinical inflammation as part of the insulin resistance syndrome: the Insulin Resistance Atherosclerosis Study (IRAS). Circulation. 2004; 102, 42
28. Devaraj S, Rogers J, Jialal I. Statins and biomarkers of inflammation. Curr Atheroscler Rep. 2007;9(1):33-41.

29. Jialal I, Stein D, Balis D, et al. Effect of hydroxymethyl glutaryl coenzyme a reductase inhibitor therapy on high sensitive C-reactive protein levels. Circulation. 2001;103 (15):1933-5.

30. Ridker PM, Danielson E, Francisco AH et al. Rosuvastatin to prevent vascular events in men and women with elevated CRP. N Engl J Med. 2008;359:2195-2207.

31. • Ridker PM, Danielson E, Francisco AH et al. Reduction in CRP and LDL-C and cardiovascular event rates after initiation of rosuvastatin : a prospective study of the JUPITER trial. Lancet. 2009;373: 1175-82. This is the first primary prevention trial showing benefit of rosuvastatin in decreasing cardiovascular events in patients with high CRP levels.

32. •• Genest J, McPherson R, Frohlich J, et al. 2009 Canadian Cardiovascular Society/Canadian guidelines for the diagnosis and treatment of dyslipidemia and prevention of cardiovascular disease in the adult: 2009 recommendations. Can J Cardiol 2009;25:567-579. These are the new guidelines from the Canadian Cardiovascular Society showing the utility of hsCRP measurements in those with intermediate risk.

33. Chrysohoou C, Panagiotakos DB, Pitsavos C, et al. Renal function, cardiovascular disease risk factors' prevalence and 5-year disease incidence; the role of diet, exercise, lipids and inflammation markers: the ATTICA study. QJM. 2010;103:413-22.

34. Wong ND, Lopez VA, L'Italien Get al. Inadequate control of hypertension in US adults with cardiovascular disease comorbidities in 2003-2004. Arch Intern Med. 2007;167:2431-6.

35. Mangat S, Agarwal S, Rosendorff C. Do statins lower blood pressure? J Cardiovasc Pharmacol Ther. 2007;12:112-23.

36. Wolfrum S, Jensen KS, Liao JK. Endothelium-dependent effects of statins. Arterioscler Thromb Vasc Biol. 2003;23:729-36.

37. Wassmann S, Faul A, Hennen B, et al. Rapid effect of 3-hydroxy3-methylglutaryl coenzyme a reductase inhibition on coronary endothelial function. Circ Res. 2003;93:e98-103.

38. Williams RR, Hunt SC, Hopkins PN, et al. Familial dyslipidemic hypertension. Evidence from 58 Utah families for a syndrome present in approximately $12 \%$ of patients with essential hypertension. JAMA. 1988;259:3579-86.

39. O'Meara JG, Kardia SL, Armon JJ, et al. Ethnic and sex differences in the prevalence, treatment, and control of dyslipidemia among hypertensive adults in the GENOA study. Arch Intern Med. 2004;164(12):1313-8.

40. Hernández-Perera $\mathrm{O}$, Pérez-Sala $\mathrm{D}$, Navarro-Antolín $\mathrm{J}$, et al. Effects of the 3-hydroxy-3-methylglutaryl-CoA reductase inhibitors, atorvastatin and simvastatin, on the expression of endothelin-1 and endothelial nitric oxide synthase in vascular endothelial cells. J Clin Invest. 1998;101:2711-9.

41. Nickenig G, Bäumer AT, Temur Y, et al. Statin-sensitive dysregulated AT1 receptor function and density in hypercholesterolemic men. Circulation. 1999;100:2131-4.

42. Verma S, Wang $\mathrm{CH}$, Li SH, et al. A self-fulfilling prophecy: Creactive protein attenuates nitric oxide production and inhibits angiogenesis. Circulation. 2002;106(8):913-9.

43. Dauphinot V, Roche F, Kossovsky MP, et al. C-reactive protein implications in new-onset hypertension in a healthy population initially aged 65 years: the Proof study. J Hypertens. 2009;27 (4):736-43.

44. Virdis A, Ghiadoni L, Plantinga Y, et al. C-reactive protein and hypertension: is there a causal relationship? Curr Pharm Des. 2007;13:1693-8.

45. Borghi C, Dormi A, Veronesi M, et al. Association between different lipid-lowering treatment strategies and blood pressure control in the Brisighella Heart Study. Am Heart J. 2004;148:28592. 
46. Strazzullo P, Kerry SM, Barbato A, et al. Do statins reduce blood pressure?: a meta-analysis of randomized, controlled trials. Hypertension. 2007;49:792-8.

47. ALLHAT Officers and Coordinators for the ALLHAT Collaborative Research Group. The Antihypertensive and Lipid-Lowering Treatment to Prevent Heart Attack Trial. Major outcomes in moderately hypercholesterolemic, hypertensive patients randomized to pravastatin vs usual care: The Antihypertensive and Lipid-Lowering Treatment to Prevent Heart Attack Trial (ALLHAT-LLT). JAMA. 2002;288:2998-3007.

48. Sever PS, Dahlöf B, Poulter NR, et al Prevention of coronary and stroke events with atorvastatin in hypertensive patients who have average or lower-than-average cholesterol concentrations, in the
Anglo-Scandinavian Cardiac Outcomes Trial-Lipid Lowering Arm (ASCOT-LLA): a multicentre randomised controlled trial. Lancet. 2003;361:1149-58.

49. King DE, Mainous AG 3rd, Egan BM, Player M, Geesey ME. Use of statins and blood pressure. Am J Hypertens. 2007;20:937-41.

50. Terzoli L, Mircoli L, Raco R, Ferrari AU. Lowering of elevated ambulatory blood pressure by HMG-CoA reductase inhibitors. J Cardiovasc Pharmacol. 2005;46:310-5.

51. •• Ridker PM, Macfayden J, Cressman M, Glynn RJ. Efficacy of Rosuvastatin among men and women with moderate CKD and elevated hsCRP. J Am Coll Cardiol 2010;55:1266-73. This is the first study to show that rosuvastatin is effective in reducing first cardiovascular events in those with CKD and elevated CRP. 